\title{
Index-Exciting CAViaR: A New Empirical Time-Varying Risk Model
}

\section{$\operatorname{AUTHOR}(\mathrm{S})$ :}

Huang, Dashan; Yu, Baimin; Lu, Zudi; Focardi, Sergio; Fukushima, Masao

\section{CITATION:}

Huang, Dashan ...[et al]. Index-Exciting CAViaR: A New Empirical Time-Varying Risk Model. STUDIES IN NONLINEAR DYNAMICS AND ECONOMETRICS 2010, 14(2): 1.

ISSUE DATE:

2010-03

URL:

http://hdl.handle.net/2433/131797

RIGHT:

(C)1999-2010 Berkeley Electronic Press 


\section{Studies in Nonlinear Dynamics \& Econometrics}

\section{Index-Exciting CAViaR: A New Empirical Time-Varying Risk Model}

Dashan Huang*

Frank J. Fabozzi**

\author{
Baimin $\mathrm{Yu}^{\dagger}$
}

Sergio Focardi ${ }^{\dagger \dagger}$
Zudi $\mathrm{Lu}^{\ddagger}$

Masao Fukushima ${ }^{\ddagger \ddagger}$

*Washington University in St. Louis, huangdas@wustl.edu

${ }^{\dagger}$ University of International Business and Economics, bmyu@amss.ac.cn

${ }^{\ddagger}$ The University of Adelaide, zudi.lu@adelaide.edu.au

**Yale School of Management, fabozzi321@aol.com

${ }^{\dagger}$ EDHEC Business School, sergio.focardi@edhec.edu

${ }_{\ddagger}^{\ddagger}$ Kyoto University, fuku@i.kyoto-u.ac.jp

Copyright (c) 2010 The Berkeley Electronic Press. All rights reserved. 


\title{
Index-Exciting CAViaR: A New Empirical Time-Varying Risk Model*
}

\author{
Dashan Huang, Baimin Yu, Zudi Lu, Frank J. Fabozzi, Sergio Focardi, and \\ Masao Fukushima
}

\begin{abstract}
Instead of assuming the distribution of return series, Engle and Manganelli (2004) propose a new Value-at-Risk (VaR) modeling approach, Conditional Autoregressive Value-at-Risk (CAViaR), to directly compute the quantile of an individual asset's returns which performs better in many cases than those that invert a return distribution. In this paper we explore more flexible CAViaR models that allow VaR prediction to depend upon a richer information set involving returns on an index. Specifically, we formulate a time-varying CAViaR model whose parameters vary according to the evolution of the index. The empirical evidence reported in this paper suggests that our timevarying CAViaR models can do a better job for VaR prediction when there are spillover effects from one market or market segment to other markets or market segments.
\end{abstract}

*We thank the editor (Bruce Mizrach) and the three anonymous referees for constructive suggestions that guided us in improving the paper. We are also grateful to Simone Manganelli for providing his CAViaR codes. Masao Fukushima was partially supported by the Scientific Research Grant-in-Aid from the Japan Society for the Promotion of Science. Zudi Lu was partially supported by a Discovery Project grant from the Australian Research Council. 


\section{Introduction}

Although the existing models for calculating Value-at-Risk (VaR) employ different methodologies, they can be generally classified into two broad categories: indirect-VaR approach and direct-VaR approach. The first category includes the classical parametric approach, nonparametric approach, and semiparametric approach, all following a common structure which can be summarized in three steps: 1) mark to market, 2) estimate the distribution of the asset return, and 3) compute VaR by inverting the distribution function. The difficulty of this category of approaches lies in the second step because financial returns usually exhibit volatility clustering (high autocorrelation), significant kurtosis (peaked and fat tailed), marginal skewness (time-varying nature) and, in the case of indexes, autocorrelation of returns. Consequently, the indirect methods for computing VaR based on the inverse distribution function of returns are usually criticized by both academic researchers and practitioners (The VaR methodology has a theoretical limitation in that it is not a coherent risk measure in the sense of Artzner et al. (1999) since it does not meet the requirement of subadditivity. However, due to its conceptual intuition, i.e., it reduces the risk associated with any kind of assets to just a number (amount in terms of a currency), which can be well understood by regulators, board members, and other interested parties, VaR is still the most extensively used analytical concept for risk management. Moreover, Danielsson et al. (2005) argue that some of the arguments against VaR may not be that important in practice).

The second category of VaR prediction, direct-VaR approach, is the dynamic quantile regression approach. This methodology does not require any assumption about the distribution of returns, but instead directly computes the quantile using regression techniques. This approach has been pioneered by Engle and Manganelli (2004). In their paper, they propose the Conditional Autoregressive VaR (CAViaR), which is based on the characteristics of volatility clustering of financial returns. (CAViaR is also a semiparametric approach from the perspective of parameter estimation.) Moreover, Manganelli and Engle demonstrate that the CAViaR model outperforms most of the indirectVaR methodologies in general when tackling fat-tailed data through Monte Carlo simulation. With respect to empirical studies, Kouretas and Zarangas (2005) employ the CAViaR model to measure the market risk of five major equity markets, six blue chip stocks from the Athens Exchange, and six major company stocks listed on the New York Stock Exchange (NYSE). Bao et al. (2006) and Kuester et al. (2006) compare CAViaR and alternative approaches for univariate VaR forecasts with different data and evaluation criteria. Table 1 summarizes the number of individual stocks and equity indexes and their 
trading location in other studies of CAViaR.

Table 1: Summary of the number of individual stocks and equity indices, and their trading location in studies of CAViaR

\begin{tabular}{|c|c|}
\hline Study & Assets \\
\hline Engle and Manganelli (2004) & $\begin{array}{l}\text { Two stocks from the New } \\
\text { York Stock Exchange and S\&P } 500\end{array}$ \\
\hline Kouretas and Zarangas (2005) & $\begin{array}{l}\text { Six stocks from the New York Stock Exchange, } \\
\text { Six stocks from the Athens Exchange, } \\
\text { CAC40, FTSE100, NIKKEI225, } \\
\text { NASDAQ, and FTSE20 }\end{array}$ \\
\hline Bao et al. (2006) & $\begin{array}{l}\text { Indonesia Jakarta Stock Exchange Composite } \\
\text { Price Index, Korea Stock Exchange Composite } \\
\text { Price Index, Malaysia Kuala Lumpur Stock, } \\
\text { Exchange Composite Index, } \\
\text { Taiwan Weight Index } \\
\text { Thailand S.E.T. Price Index }\end{array}$ \\
\hline Kuester et al. (2006) & NASDAQ Composite Index \\
\hline
\end{tabular}

The problem of model stability has been an important issue for many researchers. In practice, the model parameters are estimated from real data, which are often prone to structural changes due to regime shifts or critical social events. For example, to investigate the performance of $\mathrm{VaR}$ models for five East and Southeast Asian market indices in different environments (these markets suffered from the financial crisis of 1997-1998), Bao et al. (2006) consider three out-of-sample evaluation periods (before-crisis, crisis, and after-crisis), and find that (1) risk forecasts with CAViaR would yield poorer results during the crisis period than during tranquil periods and (2) none of the four CAViaR specifications is satisfactory in the crisis period. Because it is a dynamic regression model, the $\mathrm{CAViaR}$ model requires considerable precision of parameter estimation to describe the characteristics of the risky asset.

Based on daily return data on the NASDAQ Composite Index, Kuester et al. (2006) also provide empirical evidence (1) confirming none of the CAViaR specifications performs well overall using unconditional and conditional tests for the predictive performances of $\mathrm{VaR}$, and (2) explaining the phenomena of poor out-of-sample performance as the sample data comprise two highly volatile years, which presumably result in a deterioration of the overall performance of CAViaR. All these facts suggest that the constant-parameter CAViaR model developed by Engle and Manganelli (2004) may lose some capability when dealing with data that are subject to exogenous influence and spillover 
effects. The time-varying nature of the model parameters has been addressed in a number of papers. Venkataraman (1997) assumes the real return series follows a mixture distribution of two dummy normal distributions with binominal jumps from one distribution to another. Guidolin and Timmermann (2003), as well as Li and Lin (2004), propose a model in which the parameters change as a Markov switching process.

On the other hand, volatility spillovers play an important role in determining the model performance. Any VaR model is clearly affected by changes in the level of volatility. Volatility spillovers are the propagation of significant volatility changes from one market to another. The autoregressive nature of the original specification of CAViaR captures volatility clustering effects. However, it cannot capture volatility spillovers. In this paper, we take a different approach extending the constant-parameter CAViaR model to a new CAViaR model where the parameters of an individual risky asset are driven by the market index return. This model, which we call the index-exciting CAViaR model, allows the tails of a financial series to follow different stochastic processes and it allows VaR to be influenced by the volatility level of the driving index.

As a good complement to Engle and Manganelli (2004) and contributions that followed, the model we propose in this paper assumes the parameters are certain time-varying functions of the market index. The rationale for our model is that the magnitude and the sign of market returns might signal situations of market stress and therefore impact risk. Our model is able to accommodate different processes of tail behavior. The assumption underlying our model is that market returns are a good proxy for the local state of the market in terms of risk. Therefore, our model combines information from the volatility of the asset/portfolio which is already included in the standard CAViaR with additional information from the market state. Moreover, because of the time-varying characteristics, our model can employ one-stepahead prediction by updating the parameters immediately according to the time-varying function rather than re-estimating them. Our contribution in this paper is twofold. Our first contribution is the introduction of an alternative to the constant-parameter CAViaR model for researchers who find that the constant-parameter CAViaR may be limited. Second, we provide empirical evidence comparing the model we propose and the model proposed by Engle and Manganelli (2004).

The remainder of this paper is organized as follows. In Section 2, we provide background information about CAViaR that will be used later in the paper. An index-exciting time-varying parameter CAViaR model with three different specifications is provided in Section 3. Empirical results are provided 
in Section 4, where we also compare the predictive performances of both the constant- and time-varying parameter CAViaR models. In the final section, we provide concluding remarks. In the appendix, we review criteria for evaluating predictions made by VaR models.

\section{CAViaR}

Conceptually, VaR is defined as the maximum potential loss associated with an individual asset/portfolio for a given probability over a certain investment horizon. Mathematically, for a given significance level $\alpha$ (usually $\alpha=0.01$ or 0.05 ), let $\left\{r_{t}\right\}_{t=1}^{T}$ be a financial return series associated with one unit of an investment. Then, VaR at time $t$, conditional on the information set $\mathcal{F}_{t-1}$ ( $\sigma$-field up to time $t-1$ ), is the negative $\alpha$-quantile of the conditional return distribution, i.e.,

$$
q_{t}(\alpha)=-F_{t}^{-1}\left(\alpha \mid \mathcal{F}_{t-1}\right)=-\inf _{x}\left\{x \in \Re: P\left(r_{t} \leq x \mid \mathcal{F}_{t-1}\right)>\alpha\right\},
$$

where $F_{t}(x)=P\left(r_{t} \leq x \mid \mathcal{F}_{t-1}\right)$ denotes the conditional distribution function of the financial return series.

Typically, a VaR prediction using (1) involves the specification of $F_{t}(\cdot)$. If the dependent structure of $\left\{r_{t}\right\}$ can be fully described by a certain distribution $F_{t}(\cdot)$, VaR can be computed easily. For example, if $r_{t}=\mu_{t}+\sigma_{t} \varepsilon_{t}$ follows a normal distribution with conditional mean $\mu_{t}=E\left(r_{t} \mid \mathcal{F}_{t-1}\right)$ and conditional variance $\sigma_{t}^{2}=E\left[\left(r_{t}-\mu_{t}\right)^{2} \mid \mathcal{F}_{t-1}\right]\left(\left\{\varepsilon_{t}\right\}\right.$ is independently, identically and normally distributed (i.i.d.) with zero mean and unit variance), the prediction of VaR can be obtained by inverting the distribution function:

$$
q_{t}(\alpha)=-\left[\mu_{t}+\sigma_{t} \Phi^{-1}(\alpha)\right],
$$

where $\Phi(\cdot)$ is the standard normal distribution function.

In practice, however, most financial returns, especially short-term returns such as one day or even shorter, exhibit some untractable properties, such as volatility clustering, significant kurtosis, and marginal skewness. This was demonstrated more than 40 years ago in the pioneering work of Mandelbrot (1963) and Fama (1965). To eliminate or at least reduce the difficulties of computing VaR, Engle and Manganelli (2004), based on the fact that the volatility of financial returns is highly autocorrelated (clustering), propose a conditional autoregressive quantile approach which they refer to as CAViaR. The advantage of their approach is that it does not require any assumption about the return distribution. 
The basic idea behind CAViaR is to compute directly the conditional $\alpha$ quantile of financial returns, which can be described as

$$
q_{t}(\alpha)=\beta_{0}+\beta_{1} q_{t-1}(\alpha)+h\left(y_{t-1}, q_{t-1}(\alpha) \mid \theta\right),
$$

where $y_{t-1} \in \mathcal{F}_{t-1}, \theta$ is a parameter vector, and $h(\cdot)$ is a function linked to observable variables that belong to the information set $\mathcal{F}_{t-1}$, i.e., $h(\cdot)$ measures the impact of new information $r_{t-1}$ on VaR.

In this paper, we will focus on the following two specifications of the CAViaR model:

Symmetric CAViaR : $q_{t}(\alpha)=\beta_{0}+\beta_{1} q_{t-1}(\alpha)+\beta_{2}\left|r_{t-1}\right|$

Asymmetric CAViaR : $q_{t}(\alpha)=\beta_{0}+\beta_{1} q_{t-1}(\alpha)+\beta_{2}\left(r_{t-1}\right)^{+}+\beta_{3}\left(r_{t-1}\right)^{-}$

The characteristics and significance of the above two CAViaR specifications for practical risk management are described in Engle and Manganelli (2004), Kouretas and Zarangas (2005), and references therein. We address here that the two specifications above can accommodate different stochastic processes in the tail of financial returns and can also deal with non-i.i.d. processes.

\section{Index-Exciting CAViaR}

The motivation for developing new estimation techniques for these models is that they are the favored econometric tools for many researchers who wish to characterize co-movement in macroeconomic variables. Dynamic factor models have the advantage over observable index models, such as weighted aggregates, that one does not need to take a stand on the weighting scheme used in aggregation.

Instead of estimating the entire distribution of a financial return series, the CAViaR model computes the conditional quantile directly by simple recursive iterations, which exhibit some theoretical advantages compared with the traditional indirect approaches. However, CAViaR also faces some challenges when it is being implemented in practice. Specifically, as a regression model, CAViaR assigns the same weight to each observation and the estimation of the model's parameters may depend heavily on the length of the samples.

To improve CAViaR prediction, an alternative approach is to develop a time-varying parameter model, which has been extensively discussed in the time series literature. If volatility is influenced by other indexes, the performance of a VaR prediction model may be improved by using a richer information set involving returns on itself or an index available at the time of the 
forecast. On the other hand, the evolution of individual asset returns may be linked to the total market, i.e., the risk of an individual asset is related to the market index which tracks the performance of a specific "basket" of financial assets considered to represent a particular market or sector.

This modeling strategy takes into consideration "volatility spillover effects" which describe how a change in volatility in given markets might "spill over," that is affect other markets. The existence of volatility spillovers is described in the literature. For example, $\mathrm{Ng}$ (2000) documents how changes in volatility in the Japan and the U.S. markets propagate to six related Pacific Basin markets. From a modeling point of view, spillover effects are described by a causal relationship between those markets that drive volatility and the markets that are affected.

Therefore, adding additional information into the CAViaR model, we obtain the time-varying CAViaR model:

$$
q_{t}(\alpha)=\beta_{0}\left(\Omega_{t-1}\right)+\beta_{1}\left(\Omega_{t-1}\right) q_{t-1}(\alpha)+h\left(y_{t-1}, q_{t-1}(\alpha) \mid \theta\left(\Omega_{t-1}\right)\right),
$$

where $\Omega_{t-1}$ is the information set that consists of $\mathcal{F}_{t-1}$ and the information of market index up to time $t-1$. In this paper, the market index information represents the index return series.

Corresponding to Section 2, we consider the following two time-varying CAViaR specifications:

Time-varying symmetric CAViaR

$$
q_{t}(\alpha)=\beta_{0}\left(\gamma_{t-1}\right)+\beta_{1}\left(\gamma_{t-1}\right) q_{t-1}(\alpha)+\beta_{2}\left|r_{t-1}\right| ;
$$

Time-varying asymmetric CAViaR

$$
q_{t}(\alpha)=\beta_{0}\left(\gamma_{t-1}\right)+\beta_{1}\left(\gamma_{t-1}\right) q_{t-1}(\alpha)+\beta_{2}\left(r_{t-1}\right)^{+}+\beta_{3}\left(r_{t-1}\right)^{-}
$$

Models (2) and (3) are not specified yet because we do not know the specifications of $\beta_{0}(\cdot)$ and $\beta_{1}(\cdot)$. In the following, we consider two types of formulations on them:

Threshold time-varying specification

$$
\beta_{i}\left(\gamma_{t-1}\right)=a_{i} I\left(\left|\gamma_{t-1}\right|<\zeta\right)+b_{i} I\left(\left|\gamma_{t-1}\right| \geq \zeta\right), i=0,1
$$

Linear time-varying specification

$$
\beta_{i}\left(\gamma_{t-1}\right)=a_{i}+b_{i}\left|\gamma_{t-1}\right|, \quad i=0,1,
$$


Compared with the constant-parameter CAViaR model, the parameters to be estimated in our time-varying CAViaR specifications remain consistent and asymptotically normal. This allows us to follow Engle and Manganelli (2004) by adopting the linear and non-linear quantile techniques to estimate them; that is,

$$
\hat{\beta}=\operatorname{argmin}_{\beta \in \Re^{k}}\left\{\frac{1}{R} \sum_{t=1}^{R}\left[\alpha-I\left(r_{t}<-q_{t}(\alpha)\right)\right]\left[r_{t}+q_{t}(\alpha)\right]\right\},
$$

where $k$ is the dimension of the parameters such as $a_{i}, b_{i}, c_{i}$ (depending on the specifications of $\beta_{i}$ ) and $R$ is the size of in-samples to estimate the parameters. This regression technique has a tractable property in that it does not require any assumption about the entire distribution of the error terms. Moreover, we can easily conduct various hypothesis tests on the quantile models.

We can expect that the performance of our model will depend on the presence of spillover effects and on the correct choice of the index from which volatility "spills over." Of course, each specification of our CAViaR models with time-varying parameters requires estimating a larger number of parameters than the constant-parameter CAViaR model. Therefore, we can expect that for each sample data there is a trade off between increased model accuracy due to the time-varying coefficient and the risk of overfitting data. This issue could be resolved in-sample with criteria such as the Akaike criterion or Schwartz's Bayesian Information Criterion (BIC). In this paper, we avoid insample model selection criteria and we compare directly out-of-sample model performance.

\section{Empirical Results}

Ideally, a good model should have stable parameters over time, so that it does not need to be re-estimated very often. A model with this feature would very likely have a good performance out-of-sample, which is what practitioners are interested in. It is generally too ambitious to expect that financial models remain stable over long periods of time. A more reasonable alternative is to assume that models change slowly so that they can be estimated over a moving window of sufficient length and eventually be reused for some time before parameters need to be re-estimated. In our test, we estimate and test models over the period $11 / 7 / 1994$ to $9 / 30 / 2008$. In this period there are 3,500 daily returns. We use moving windows 1,000 days long (i.e., four years), and we keep models constant for the following 250 days (i.e., one year) before re- 
estimating. Therefore, in total, we estimate models 10 times in the period considered.

Our dataset is formed by six indexes available on the website of Kenneth French constructed based on size (market capitalization) and book-to-market value ratio. The exciting index is the value-weighted return on all NYSE, AMEX, and NASDAQ stocks, also available on Kenneth French's website.

Clearly we cannot expect that the six indexes be all equally influenced by the index. Therefore, we can expect that performance will vary from index to index. For testing performance, we use the framework set forth in Berkowitz, Christoffersen, and Pelletier (2009).

In our dataset, the tail percentile of the $\mathrm{VaR}$ prediction is set at $\theta=1 \%$ and $5 \%$, which yield one-sided $99 \%$ and $95 \%$ VaRs. This is quite far in the tail but is typical of the VaR forecasts used at banks (Berkowitz and O'Brien, 2002)

Violations of the VaR should occur randomly over time and should not be clustered over time. If it can be predicted that volatility will be increasing in the near future, then the model used to compute the VaR should take this information into account and adjust the VaR accordingly. In other words, if the model used to compute VaR is correctly specified, then violations should only happen because of unpredictable events.

Under the 1996 Market Risk Amendment to the Basle Accord effective in 1998, qualifying financial institutions have the freedom to specify their own model to compute their VaR. It thus becomes crucially important for regulators to assess the quality of the models employed by assessing the forecast accuracy - a procedure known as backtesting. The accuracy of a set of VaR forecasts can be assessed by viewing them as one-sided forecasts. A violation of VaR is defined as occurring when the ex post return is lower than the VaR.

In our test we compare the performance of the constant-parameter CAViaR model with our index-exciting CAViaR model. As expected, the results are mixed. Our explanation is that the different indexes we consider are subject to different spillover effects. When spillover effects are not significant, the additional parameterization of the index-exciting CAViaR model penalizes performance. Our results are summarized in Tables 2-7.

Following the Berkowitz, Christoffersen, and Pelletier framework, performance is evaluated along the dimensions of conditional coverage and independence tests. It should be kept in mind that VaR violations are rare events especially with $\alpha=0.01$ on the average.

Tables 2-7 present p-values associated with the tests. Setting the confidence level at $95 \%$, as typically done, models where p-values are systematically below 0.05 have to be considered problematic. From the tables, we can see that 
our model outperforms the standard CAViaR model in cases where portfolios are formed with small cap stocks or large cap with low book-to-marketratios stocks. This is reasonable because small cap stocks are those most subject to spillover effects. Figures 1-4 exhibit the evolution of $\beta_{0}$ and $\beta_{1}$ of the ASand SAV-CAViaR models at the 1\% level for the small capitalization and low book-to-market ratio stocks (figures for other cases are available upon request). Clearly, our time-varying CAViaR models have different parameters with the constant-CAViaR models. This can readjust the VaR prediction more quickly and flexibly in response to vagaries in the market risk.

\section{Conclusion}

In this paper, we developed an index-exciting parameter CAViaR model whose parameters vary according to the evolution of the index. The empirical evidence reported in this paper suggests that our time-varying CAViaR models can do a better job of VaR prediction when there are spillover effects from one market or market segment to other markets or market segments. 
Table 2: Portfolio formed with small capitalization and low book-to-market ratio stocks

\begin{tabular}{|c|c|c|c|c|c|c|c|c|c|c|}
\hline CAViaR & $\hat{\alpha}$ & $\hat{Q}$ & $D Q_{H i t}$ & $\begin{array}{r}D Q_{V a R} \\
\text { SAV }\end{array}$ & $\begin{array}{l}\text { p-LRuc } \\
1 \%\end{array}$ & p-LRind & p-LRcc & p-LB1 & p-LB5 & p-Lrduration \\
\hline Constant & 0.0116 & 0.0411 & 0.0000 & 0.0000 & 0.4330 & 0.4092 & 0.5231 & 0.5569 & 0.0000 & 0.0025 \\
\hline Threshold & 0.0152 & 0.0443 & 0.0000 & 0.0000 & 0.0152 & 0.6086 & 0.0461 & 0.5725 & 0.0000 & 0.0000 \\
\hline Linear & 0.0160 & 0.1101 & 0.0000 & $\begin{array}{c}0.0000 \\
\text { SAV }\end{array}$ & $\begin{array}{l}0.0055 \\
5 \%\end{array}$ & 0.2600 & 0.0113 & 0.4275 & 0.0000 & 0.0000 \\
\hline Constant & 0.0504 & 0.1465 & 0.1301 & 0.0117 & 0.9270 & 0.8818 & 0.9849 & 0.8827 & 0.1327 & 0.9989 \\
\hline Threshold & 0.0540 & 0.1543 & 0.0014 & 0.0000 & 0.3647 & 0.0886 & 0.1556 & 0.0652 & 0.0012 & 0.0378 \\
\hline Linear & 0.0556 & 0.1494 & 0.0005 & $\begin{array}{r}0.0001 \\
\text { AS }\end{array}$ & $\begin{array}{l}0.2066 \\
1 \%\end{array}$ & 0.2386 & 0.2249 & 0.2129 & 0.0008 & 0.2366 \\
\hline Constant & 0.0216 & 0.0432 & 0.0000 & 0.0000 & 0.0000 & 0.1225 & 0.0000 & 0.2692 & 0.0001 & 0.0000 \\
\hline Threshold & 0.0204 & 0.0434 & 0.0000 & 0.0000 & 0.0000 & 0.1449 & 0.0000 & 0.2973 & $0.0360^{*}$ & 0.0000 \\
\hline Linear & 0.0284 & 0.1851 & 0.0000 & $\begin{array}{r}0.0000 \\
\text { AS }\end{array}$ & $5 \%$ & 0.0430 & 0.0000 & 0.1492 & 0.0000 & 0.0000 \\
\hline Constant & 0.0640 & 0.1497 & 0.0013 & 0.0000 & 0.0020 & 0.6723 & 0.0079 & 0.6777 & 0.1808 & 0.0129 \\
\hline Threshold & 0.0668 & 0.1516 & 0.0003 & 0.0000 & 0.0002 & 0.1554 & 0.0004 & 0.1817 & 0.2802 & 0.0022 \\
\hline Linear & 0.0616 & 0.1503 & $0.0114^{*}$ & 0.0000 & $0.0101^{*}$ & 0.1988 & $0.0160^{*}$ & 0.2269 & 0.2603 & $0.0525^{* *}$ \\
\hline
\end{tabular}

Note: In our test, we use the data from Ken French website and test models over the period 11/7/1994 to 9/30/2008. In this period there are 3,500 daily returns. We use moving windows 1,000 days long and keep models constant for the following 250 days before re-estimating. $\hat{\alpha}$ is the empirical violation of VaR prediction (Eq: (6)), $\hat{Q}$ is the empirical loss (Eq: (7)), $D Q_{H i t}$ and $D Q_{V a R}$ is the dynamic quantile test without and with VaR lagged terms (Eq: (11)), p-LRuc is the unconditional coverage test (Eq: (5)), p-LRind is the independent test (Eq: (8)), p-LRcc is the conditional coverage test (Eq: (9)), p-LB1 is the autocorrelation test with lags 1 (Eq: (12)), p-LB5 is the autocorrelation test with lags 5 (Eq: (12)), p-Lrduration is the duration test (Eq: (13)). "Constant" represents the constant-parameter CAViaR model, "Threshold" represents the index-exciting threshold CAViaR model, "Linear" represents the index-exciting linear CAViaR model, SAV represents the symmetric CAViaR model, AS represents asymmetric CAViaR model. * means our model is not significant whereas the constant-parameter CAViaR model is significant at the $1 \%$ level. ** means our model is not significant whereas the constant-parameter CAViaR model is significant at the $5 \%$ level. 
Table 3: Portfolio formed with small capitalization and median book-to-market ratio stocks

\begin{tabular}{|c|c|c|c|c|c|c|c|c|c|c|}
\hline CAViaR & $\hat{\alpha}$ & $\hat{Q}$ & $D Q_{H i t}$ & $\begin{array}{r}D Q_{V a R} \\
\text { SAV }\end{array}$ & $\begin{array}{l}\text { p-LRuc } \\
1 \%\end{array}$ & p-LRind & p-LRcc & p-LB1 & p-LB5 & p-Lrduration \\
\hline Constant & 0.0172 & 0.0338 & 0.0000 & 0.0000 & 0.0010 & 0.2198 & 0.0022 & 0.3811 & 0.0000 & 0.0003 \\
\hline Threshold & 0.0108 & 0.0318 & $0.6830^{* *}$ & $0.3439^{* *}$ & $0.6915^{* *}$ & 0.4425 & $0.6882^{* *}$ & 0.5848 & $0.4821^{* *}$ & $0.5193^{* *}$ \\
\hline Linear & 0.0092 & 0.0310 & $0.3249^{* *}$ & $\begin{array}{r}0.1427^{* *} \\
\text { SAV }\end{array}$ & $\begin{array}{l}0.6836^{* *} \\
5 \%\end{array}$ & 0.5133 & $0.7433^{* *}$ & 0.6421 & $0.0933^{* *}$ & $0.2616^{* *}$ \\
\hline Constant & 0.0576 & 0.1201 & 0.1095 & 0.0044 & 0.0883 & 0.6242 & 0.2076 & 0.6323 & 0.3956 & 0.2877 \\
\hline Threshold & 0.0672 & 0.1251 & 0.0000 & 0.0000 & 0.0002 & 0.8234 & 0.0008 & 0.8217 & 0.0037 & 0.0003 \\
\hline Linear & 0.0640 & 0.1195 & 0.0004 & $\begin{array}{r}0.0000 \\
\mathrm{AS}\end{array}$ & $\begin{array}{l}0.0020 \\
1 \%\end{array}$ & 0.6723 & 0.0079 & 0.6777 & 0.0536 & 0.0128 \\
\hline Constant & 0.0248 & 0.0362 & 0.0000 & 0.0000 & 0.0000 & 0.6354 & 0.0000 & 0.6561 & 0.0225 & 0.0000 \\
\hline Threshold & 0.0192 & 0.0333 & 0.0001 & 0.0000 & 0.0000 & 0.9348 & 0.0002 & 0.9339 & 0.0185 & 0.0000 \\
\hline Linear & 0.0208 & 0.0318 & 0.0000 & $\begin{array}{r}0.0000 \\
\text { AS }\end{array}$ & $\begin{array}{l}0.0000 \\
5 \%\end{array}$ & 0.9350 & 0.0000 & 0.9358 & 0.0036 & 0.0000 \\
\hline Constant & 0.0720 & 0.1204 & 0.0000 & 0.0000 & 0.0000 & 0.3578 & 0.0000 & 0.3745 & 0.0837 & 0.0000 \\
\hline Threshold & 0.0648 & 0.1178 & 0.0012 & 0.0001 & 0.0011 & 0.1096 & 0.0014 & 0.1371 & 0.2087 & 0.0082 \\
\hline Linear & 0.0676 & 0.1195 & 0.0000 & 0.0000 & 0.0001 & 0.0610 & 0.0001 & 0.0848 & 0.0254 & 0.0012 \\
\hline
\end{tabular}

Note: The notation is provided in Table 2. 
Table 4: Portfolio formed with small capitalization and high book-to-market ratio stocks

\begin{tabular}{|c|c|c|c|c|c|c|c|c|c|c|}
\hline CAViaR & $\hat{\alpha}$ & $\hat{Q}$ & $D Q_{H i t}$ & $\begin{array}{r}D Q_{V a R} \\
\mathrm{SAV}\end{array}$ & $\begin{array}{l}\text { p-LRuc } \\
1 \%\end{array}$ & p-LRind & p-LRcc & p-LB1 & p-LB5 & p-Lrduration \\
\hline Constant & 0.0168 & 0.0353 & 0.0000 & 0.0000 & 0.0018 & 0.2308 & 0.0038 & 0.3924 & 0.0007 & 0.0006 \\
\hline Threshold & 0.0168 & 0.0360 & 0.0000 & 0.0000 & 0.0018 & 0.2308 & 0.0038 & 0.3924 & 0.0000 & 0.0007 \\
\hline Linear & 0.0156 & 0.0345 & 0.0000 & $\begin{array}{r}0.0000 \\
\text { SAV }\end{array}$ & $\begin{array}{l}0.0093 \\
5 \%\end{array}$ & 0.2661 & $0.0183^{*}$ & 0.4277 & 0.0001 & 0.0009 \\
\hline Constant & 0.0600 & 0.1137 & 0.1328 & 0.1614 & 0.0259 & 0.4922 & 0.0661 & 0.4787 & 0.7413 & 0.1096 \\
\hline Threshold & 0.0692 & 0.1180 & 0.0000 & 0.0000 & 0.0000 & 0.0786 & 0.0000 & 0.0613 & 0.0335 & 0.0002 \\
\hline Linear & 0.0668 & 0.1160 & 0.0000 & $\begin{array}{r}0.0000 \\
\text { AS }\end{array}$ & $\begin{array}{l}0.0002 \\
1 \%\end{array}$ & 0.0414 & 0.0001 & 0.0281 & 0.0297 & 0.0016 \\
\hline Constant & 0.0264 & 0.0400 & 0.0000 & 0.0000 & 0.0000 & 0.5311 & 0.0000 & 0.5629 & 0.0001 & 0.0000 \\
\hline Threshold & 0.0324 & 0.4340 & 0.0000 & 0.0000 & 0.0000 & 0.0000 & 0.0000 & 0.0000 & 0.0000 & 0.0000 \\
\hline Linear & 0.0240 & 0.0393 & 0.0000 & $\begin{array}{r}0.0000 \\
\mathrm{AS}\end{array}$ & $\begin{array}{l}0.0000 \\
5 \%\end{array}$ & 0.6512 & 0.0000 & 0.6327 & 0.0002 & 0.0000 \\
\hline Constant & 0.0728 & 0.1146 & 0.0000 & 0.0000 & 0.0000 & 0.7062 & 0.0000 & 0.7099 & 0.2441 & 0.0000 \\
\hline Threshold & 0.0708 & 0.1130 & 0.0000 & 0.0000 & 0.0000 & 0.0694 & 0.0000 & 0.0921 & 0.2094 & 0.0000 \\
\hline Linear & 0.0732 & 0.1218 & 0.0000 & 0.0000 & 0.0000 & 0.6428 & 0.0000 & 0.6372 & 0.0155 & 0.0000 \\
\hline
\end{tabular}

Note: The notation is provided in Table 2. 
Table 5: Portfolio formed with large capitalization and low book-to-market ratio stocks

\begin{tabular}{|c|c|c|c|c|c|c|c|c|c|c|}
\hline CAViaR & $\hat{\alpha}$ & $\hat{Q}$ & $D Q_{H i t}$ & $\begin{array}{r}D Q_{V a R} \\
\mathrm{SA}\end{array}$ & $\begin{array}{l}\text { p-LRuc } \\
1 \%\end{array}$ & p-LRind & p-LRcc & p-LB1 & p-LB5 & p-Lrduration \\
\hline Constant & 0.0168 & 0.0370 & 0.0000 & 0.0000 & 0.0018 & 0.7372 & 0.0074 & 0.7216 & 0.0000 & 0.0000 \\
\hline Threshold & 0.0200 & 0.0393 & 0.0000 & 0.0000 & 0.0000 & 0.0968 & 0.0000 & 0.0412 & 0.0000 & 0.0000 \\
\hline Linear & 0.0160 & 0.0369 & 0.0000 & $\begin{array}{r}0.0000 \\
\mathrm{SA}\end{array}$ & $\begin{array}{l}0.0055 \\
5 \%\end{array}$ & 0.6723 & $0.0195^{*}$ & 0.6475 & 0.0000 & 0.0000 \\
\hline Constant & 0.0868 & 0.2874 & 0.0000 & 0.0000 & 0.0000 & 0.0000 & 0.0000 & 0.0000 & 0.0000 & 0.0000 \\
\hline Threshold & 0.0624 & 0.5897 & 0.0000 & 0.0000 & 0.0061 & 0.0000 & 0.0000 & 0.0000 & 0.0000 & 0.0000 \\
\hline Linear & 0.0428 & 1.3374 & 0.0000 & $\begin{array}{r}0.0000 \\
\mathrm{~A}\end{array}$ & $\begin{array}{l}0.0907^{* *} \\
1 \%\end{array}$ & $0.2703^{* *}$ & $0.1302^{* *}$ & $0.2374^{* *}$ & 0.0000 & 0.0000 \\
\hline Constant & 0.0176 & 0.0382 & 0.0000 & 0.0000 & 0.0006 & 0.2092 & 0.0012 & 0.3699 & 0.0032 & 0.0001 \\
\hline Threshold & 0.0204 & 0.0387 & 0.0000 & 0.0000 & 0.0000 & 0.3923 & 0.0000 & 0.3368 & 0.0004 & 0.0000 \\
\hline Linear & 0.0200 & 0.0375 & 0.0000 & $\begin{array}{r}0.0000 \\
\mathrm{~A}\end{array}$ & $\begin{array}{l}0.0000 \\
5 \%\end{array}$ & 0.9997 & 0.0001 & 0.9997 & $0.0641^{* *}$ & 0.0000 \\
\hline Constant & 0.0652 & 0.1282 & 0.0000 & 0.0000 & 0.0008 & 0.3691 & 0.0025 & 0.3875 & 0.0004 & 0.0000 \\
\hline Threshold & 0.0484 & 0.1280 & $0.0690^{* *}$ & 0.0096 & $0.7122^{* *}$ & 0.1743 & $0.3712^{* *}$ & 0.2142 & $0.0729^{* *}$ & 0.0034 \\
\hline Linear & 0.0532 & 0.1250 & 0.0026 & 0.0005 & $0.4673^{* *}$ & 0.6610 & $0.6974^{* *}$ & 0.6683 & 0.0010 & 0.0020 \\
\hline
\end{tabular}

Note: The notation is provided in Table 2. 
Table 6: Portfolio formed with large capitalization and median book-to-market ratio stocks

\begin{tabular}{|c|c|c|c|c|c|c|c|c|c|c|}
\hline CAViaR & $\hat{\alpha}$ & $\hat{Q}$ & $D Q_{H i t}$ & $\begin{array}{r}D Q_{V a R} \\
\text { SAV }\end{array}$ & $\begin{array}{l}\text { p-LRuc } \\
1 \%\end{array}$ & p-LRind & p-LRcc & p-LB1 & p-LB5 & p-Lrduration \\
\hline Constant & 0.0140 & 0.0358 & 0.0000 & 0.0000 & 0.0580 & 0.3187 & 0.1009 & 0.4773 & 0.0000 & 0.0007 \\
\hline Threshold & 0.0208 & 0.0376 & 0.0000 & 0.0000 & 0.0000 & 0.1371 & 0.0000 & 0.2877 & 0.0007 & 0.0000 \\
\hline Linear & 0.0156 & 0.0372 & 0.0000 & $\begin{array}{r}0.0000 \\
\text { SAV }\end{array}$ & $\begin{array}{l}0.0093 \\
5 \%\end{array}$ & 0.2661 & 0.0183 & 0.4277 & 0.0002 & 0.0035 \\
\hline Constant & 0.0592 & 0.1175 & 0.0019 & 0.0041 & 0.0400 & 0.6637 & 0.1104 & 0.6573 & 0.0197 & 0.0775 \\
\hline Threshold & 0.0736 & 0.1278 & 0.0000 & 0.0000 & 0.0000 & 0.0406 & 0.0000 & 0.0287 & 0.0000 & 0.0000 \\
\hline Linear & 0.0668 & 0.1392 & 0.0000 & $\begin{array}{r}0.0000 \\
\text { AS }\end{array}$ & $\begin{array}{l}0.0002 \\
1 \%\end{array}$ & 0.0788 & 0.0002 & 0.0608 & 0.0000 & 0.0000 \\
\hline Constant & 0.0148 & 0.0348 & 0.0001 & 0.0001 & 0.0244 & 0.2916 & 0.0455 & 0.4521 & 0.0199 & 0.0090 \\
\hline Threshold & 0.0176 & 0.0358 & 0.0000 & 0.0000 & 0.0006 & 0.2092 & 0.0012 & 0.3699 & 0.0003 & 0.0000 \\
\hline Linear & 0.0192 & 0.0360 & 0.0000 & $\begin{array}{r}0.0000 \\
\mathrm{AS}\end{array}$ & $\begin{array}{l}0.0000 \\
5 \%\end{array}$ & 0.9348 & 0.0002 & 0.9339 & 0.0007 & 0.0000 \\
\hline Constant & 0.0588 & 0.1181 & 0.2325 & 0.0789 & 0.0492 & 0.8131 & 0.1406 & 0.8150 & 0.7957 & 0.1253 \\
\hline Threshold & 0.0620 & 0.1208 & 0.0103 & 0.0000 & 0.0079 & 0.6404 & 0.0262 & 0.6334 & 0.2365 & 0.0046 \\
\hline Linear & 0.0620 & 0.1192 & 0.0138 & 0.0020 & 0.0079 & 0.8950 & 0.0290 & 0.8943 & 0.3623 & 0.0153 \\
\hline
\end{tabular}

Note: The notation is provided in Table 2. 
Table 7: Portfolio formed with large capitalization and high book-to-market ratio stocks

\begin{tabular}{|c|c|c|c|c|c|c|c|c|c|c|}
\hline CAViaR & $\hat{\alpha}$ & $\hat{Q}$ & $D Q_{H i t}$ & $\begin{array}{r}D Q_{V a R} \\
\mathrm{SAV}\end{array}$ & $\begin{array}{l}\text { p-LRuc } \\
1 \%\end{array}$ & p-LRind & p-LRcc & p-LB1 & p-LB5 & p-Lrduration \\
\hline Constant & 0.0140 & 0.0351 & 0.0023 & 0.0047 & 0.0580 & 0.3187 & 0.1009 & 0.4773 & 0.0471 & 0.0263 \\
\hline Threshold & 0.0140 & 0.0406 & 0.0000 & 0.0000 & 0.0580 & 0.3187 & 0.1009 & 0.4773 & 0.0002 & 0.0002 \\
\hline Linear & 0.0172 & 0.0412 & 0.0000 & $\begin{array}{l}0.0000 \\
\text { SAV }\end{array}$ & $\begin{array}{l}0.0010 \\
5 \%\end{array}$ & 0.0422 & 0.0006 & 0.0075 & 0.0000 & 0.0000 \\
\hline Constant & 0.0580 & 0.1248 & 0.0003 & 0.0005 & 0.0732 & 0.1167 & 0.0587 & 0.0930 & 0.0006 & 0.0007 \\
\hline Threshold & 0.0812 & 0.2298 & 0.0000 & 0.0000 & 0.0000 & 0.0009 & 0.0000 & 0.0003 & 0.0000 & 0.0000 \\
\hline Linear & 0.0828 & 0.2603 & 0.0000 & $\begin{array}{r}0.0000 \\
\mathrm{AS}\end{array}$ & $\begin{array}{l}0.0000 \\
1 \%\end{array}$ & 0.0018 & 0.0000 & 0.0007 & 0.0000 & 0.0000 \\
\hline Constant & 0.0200 & 0.0392 & 0.0000 & 0.0000 & 0.0000 & 0.1530 & 0.0000 & 0.3071 & 0.1287 & 0.0000 \\
\hline Threshold & 0.0236 & 0.0412 & 0.0000 & 0.0000 & 0.0000 & 0.7200 & 0.0000 & 0.7329 & 0.3215 & 0.0000 \\
\hline Linear & 0.0192 & 0.0362 & 0.0001 & $\begin{array}{r}0.0000 \\
\mathrm{AS}\end{array}$ & $\begin{array}{l}0.0000 \\
5 \%\end{array}$ & 0.9348 & 0.0002 & 0.9339 & 0.6081 & 0.0001 \\
\hline Constant & 0.0660 & 0.1346 & 0.0034 & 0.0004 & 0.0005 & 0.7690 & 0.0020 & 0.7716 & 0.5329 & 0.0008 \\
\hline Threshold & 0.0720 & 0.1365 & 0.0000 & 0.0000 & 0.0000 & 0.7703 & 0.0000 & 0.7725 & 0.0671 & 0.0000 \\
\hline Linear & 0.0736 & 0.1254 & 0.0000 & 0.0000 & 0.0000 & 0.8950 & 0.0000 & 0.8944 & 0.0391 & 0.0000 \\
\hline
\end{tabular}

Note: The notation is provided in Table 2. 

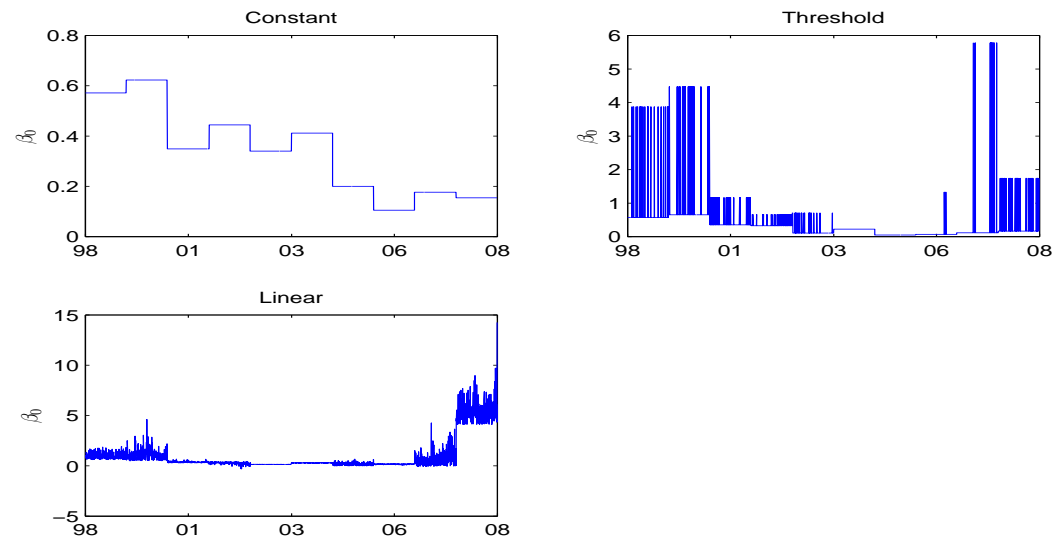

Figure 1: Evolution of $\beta_{0}$ of the AS-CAViaR model at the $1 \%$ level for the small capitalization and low book-to-market ratio stocks
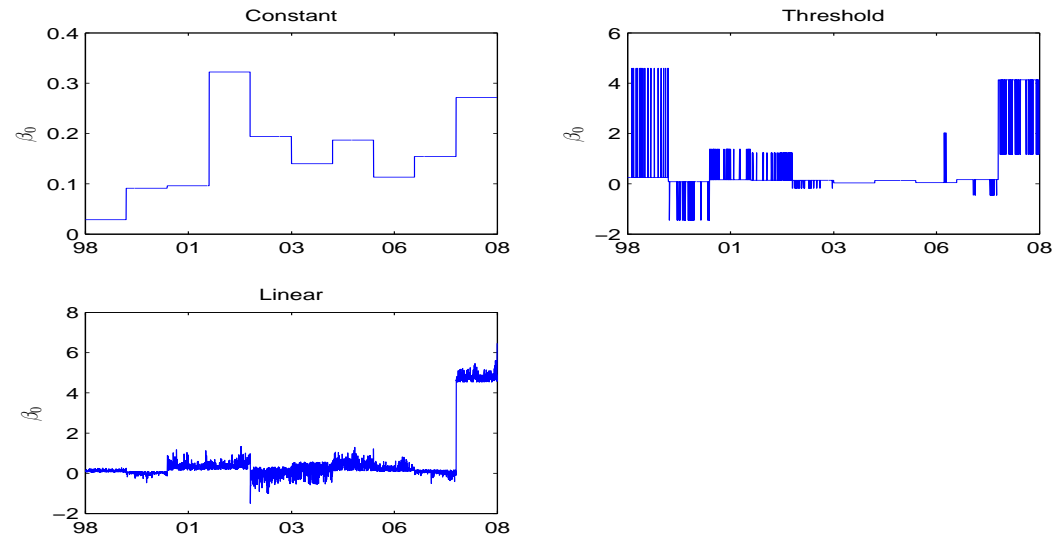

Figure 2: Evolution of $\beta_{0}$ of the SAV-CAViaR model at the $1 \%$ level for the small capitalization and low book-to-market ratio stocks 

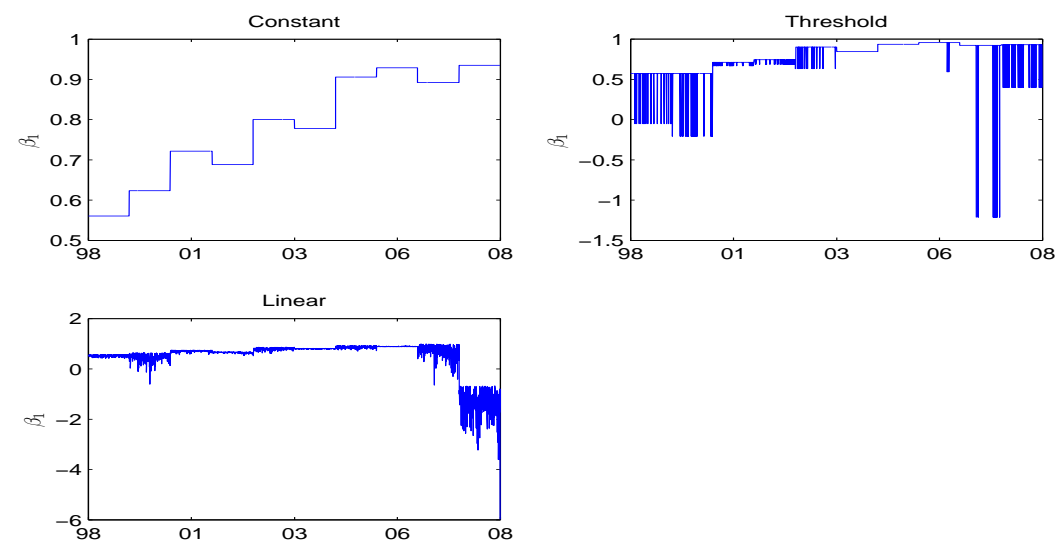

Figure 3: Evolution of $\beta_{1}$ of the AS-CAViaR model at the $1 \%$ level for the small capitalization and low book-to-market ratio stocks
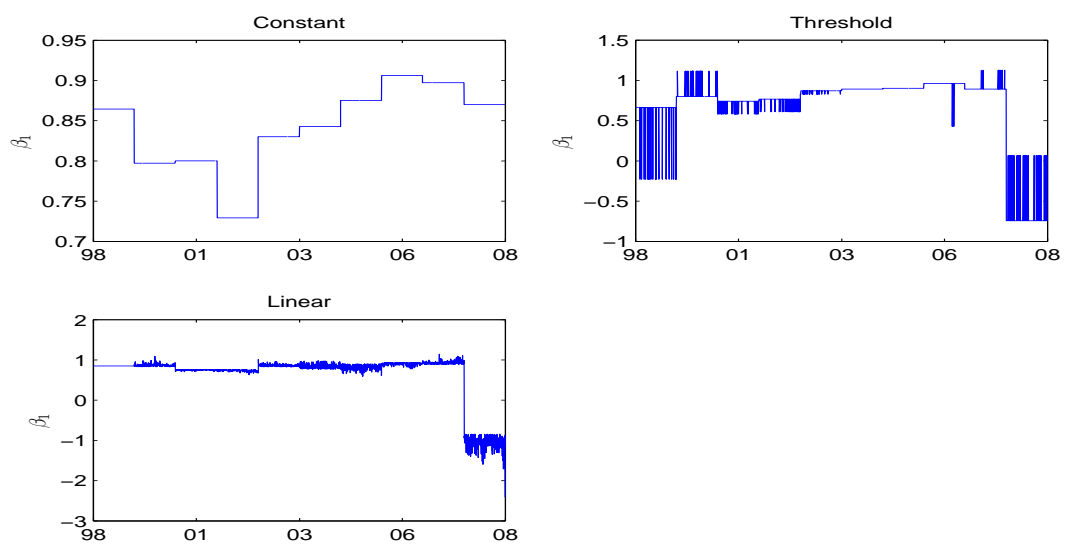

Figure 4: Evolution of $\beta_{1}$ of the SAV-CAViaR model at the $1 \%$ level for the small capitalization and low book-to-market ratio stocks 


\section{Appendix: Evaluation of Performance}

To assess the predictive performance of the proposed models, in this appendix we explain the tests we employ for evaluating the predictive quantile performance.

When assessing the accuracy of a VaR prediction model, there is always a general hypothesis required to be satisfied. That is,

$$
\mathbb{E}\left[H_{t}(\alpha) \mid \mathcal{F}_{t-1}\right]=\alpha,
$$

where $H_{t}(\alpha)=I\left(r_{t}<-q_{t}(\alpha)\right)$. If (4) holds, we say the VaR prediction is efficient with respect to $\mathcal{F}_{t-1}$. This means $H_{t}(\alpha)$ is uncorrelated with any information in $\mathcal{F}_{t-1}$ up to time $t-1$. In this case, the forecast for $\mathrm{VaR}_{t}$ and $H_{t}(\alpha)$ cannot be improved. There are two implications involved in condition (4): correct unconditional coverage and independence of VaR forecasts (Christoffersen, 1998; Danielsson and Morimoto, 2000; Bertail et al., 2004; and Komunjer, 2004).

\section{Unconditional coverage test}

Because different methods of computing VaR tend to produce different results and it is unlikely that estimates from a model will exhibit all the properties of accurate forecasts for different risky assets at different significant levels, evaluating the accuracy of the models underlying them is necessarily indispensable for supervisors and regulators. Up to now, many types of criteria and methods have been developed to assess VaR estimates. In this paper, we use the proportion of failure test proposed by Kupiec (1995). Suppose VaR estimates are accurate. Then, the event of failure $H_{t}(\alpha)=1$ can be modeled as independent draws from a binomial distribution with a probability of occurrence equal to the significance level, that is, $\hat{\alpha}=\alpha$. Thus, the likelihood-ratio test statistic is

$$
\mathrm{LR}_{u c}=-2 \ln \left[(1-\alpha)^{n-m} \alpha^{m}\right]+2 \ln \left[(1-\hat{\alpha})^{n-m} \hat{\alpha}^{m}\right] \sim \chi^{2}(1),
$$

where $m$ is the number of violations and

$$
\hat{\alpha}=m / n=\frac{1}{n} \sum_{t=R+1}^{T} \hat{H}_{t}(\alpha) .
$$

At the same time, we can also compute the empirical expected loss of $\mathrm{VaR}_{\mathrm{t}}$ as

$$
\hat{Q}(\alpha)=\frac{1}{n} \sum_{t=1}^{n}\left[\alpha-\hat{H}_{t}\right]\left[r_{t}+\mathrm{VâR}_{t}\right]
$$


The main goal of this test is to examine whether the failure rate of a model is statistically equal to the expected one and therefore to ensure that a financial institution will not mis-allocate its capital.

\section{Independence test}

The unconditional coverage test can reject a model for both high and low VaR violations but its power is generally poor especially for high confidence levels. Actually, a good VaR model must generate independent forecasts, that is, a violation $\left(H_{t}(\alpha)=1\right)$ today has no effect on the probability of a violation tomorrow.

Christoffersen (1998) formulates the test ratio of independence through a first-order Markov-chain, which is given as a transition matrix

$$
\Pi=\left(\begin{array}{ll}
1-\pi_{01} & \pi_{01} \\
1-\pi_{11} & \pi_{11}
\end{array}\right)
$$

where $\pi_{i j}=P\left(H_{t}(\alpha)=j \mid H_{t-1}(\alpha)=i\right), i$ and $j$ refer to states of violation/nonviolatons.

Under the hypothesis of independence, the test statistic takes the form

$$
\mathrm{LR}_{\text {ind }}=2 \ln \left[\frac{\left(1-\hat{\pi}_{01}\right)^{n_{00}} \hat{\pi}_{01}^{n_{01}}\left(1-\hat{\pi}_{11}\right)^{n_{10}} \hat{\pi}_{11}^{n_{11}}}{(1-\hat{\pi})^{n_{00}+n_{10}} \hat{\pi}^{n_{01}+n_{11}}}\right] \sim \chi^{2}(1)
$$

where $n_{i j}$ indicates the number of value $j$ following $i$ in the VaR violation sequence, and

$$
\begin{aligned}
\hat{\pi}_{01} & =\frac{n_{01}}{n_{00}+n_{01}}, \\
\hat{\pi}_{11} & =\frac{n_{11}}{n_{10}+n_{11}}, \\
\hat{\pi} & =\frac{n_{01}+n_{11}}{n_{00}+n_{01}+n_{10}+n_{11}},
\end{aligned}
$$

\section{Conditional coverage test}

Unconditional coverage and independence are fundamental properties of an adequate VaR model, and they can be formalized into a joint property which is know as conditional coverage, a stronger condition than the previous ones.

Christoffersen (1998) defines a VaR violation sequence to have correct conditional coverage if the violation sequence is identically and independently 
distributed and proposes the following likelihood ratio test that combines the test statistic of unconditional coverage and the test statistic of independence:

$$
\mathrm{LR}_{c c}=\mathrm{LR}_{u c}+\mathrm{LR}_{\text {ind }} \sim \chi^{2}(2)
$$

The discussion of joint tests so far might seem to suggest that joint tests are universally preferable to tests of either the unconditional coverage property or independence property alone. While joint tests have the property that they will eventually detect a VaR measure which violates either of these properties, this comes at the expense of a decreased ability to detect a VaR measure which only violates one of the two properties. If, for example, a VaR measure exhibits appropriate unconditional coverage but violates the independence property then an independence test has a greater likelihood of detecting this inaccurate VaR measure than a joint test. The joint test is hampered by the fact that one of the two violations it is designed to detect, namely violations of unconditional coverage, is actually satisfied by the VaR measure. The fact that one of the two properties is satisfied makes it more difficult for the joint test to detect the inadequacy of the VaR measure.

Campbell (2005) analyzes the reduced power of the test of conditional coverage against separate tests of unconditional coverage and independence. Separate tests can have better power to detect a VaR model that violates a specific property, while the joint test of conditional coverage has decreased ability to detect a model that violates only one of the two conditions and satisfies the other. The joint test of conditional coverage has a broader scope of detection but at the loss of some statistical power.

\section{Dynamic quantile test}

Christoffersen's Markov-chain in the independence test has some limitation because it only considers the first-order dependence in the $\left\{H_{t}(\alpha)\right\}$ series. Obviously, it would fail to reject an $\left\{H_{t}(\alpha)\right\}$ series that does not have firstorder Markov dependence but does exhibit some higher-order dependences and other backtesting factors.

Engle and Manganelli (2004) propose a linear regression test to test the conditional efficiency hypothesis where the dependent variable is the de-meaned indicator function at time $t, H_{t}(\alpha)-\alpha$, and the independent variables are the previous elements of the de-meaned VaR violation sequence and the chosen exogenous variables. Mathematically, this test investigates the regression of 
$H_{t}(\alpha)$ on $H_{t-i}(\alpha)(i=1, \cdots, M)$ and $q_{t}(\alpha)$; that is,

$$
H_{t}(\alpha)=\alpha_{0}+\sum_{i=1}^{M} w_{i} H_{t-i}(\alpha)+w_{M+1} q_{t}(\alpha)+u_{t},
$$

where $w_{i}, i=1, \cdots, M+1$, are regression parameters and

$$
u_{t}=\left\{\begin{array}{l}
-\alpha, \text { with probability } 1-\alpha \\
1-\alpha, \text { with probability } \alpha
\end{array}\right.
$$

Under the null hypothesis, $\alpha_{0}=\alpha$ and $w_{i}=0, i=1, \cdots, M+1$. Denoting $w_{0}=\alpha_{0}-\alpha$, we rewrite (10) in vector notation as

$$
\mathbf{H}-\alpha \mathbf{1}=\mathbf{X} \boldsymbol{w}+\mathbf{u}
$$

where 1 denotes the vector of ones and

$$
\mathbf{X}=\left(\begin{array}{cccccc}
1 & H_{M} & H_{M-1} & \cdots & H_{1} & q_{M+1} \\
1 & H_{M+1} & H_{M} & \cdots & H_{2} & q_{M+2} \\
\cdots & \cdots & \cdots & \cdots & \cdots & \cdots \\
1 & H_{n-1} & H_{n-2} & \cdots & H_{n-M} & q_{n}
\end{array}\right)
$$

Thus, under the null hypothesis, $\boldsymbol{w}=0$, Engle and Manganelli (2004) prove

$$
\hat{\boldsymbol{w}}=\left(\mathbf{X}^{\prime} \mathbf{X}\right)^{-1} \mathbf{X}^{\prime}(\mathbf{H}-\alpha \mathbf{1}) \sim \mathcal{N}\left(0,\left(\mathbf{X}^{\prime} \mathbf{X}\right)^{-1} \alpha(1-\alpha)\right),
$$

and hence propose the following test statistic:

$$
\mathrm{DQ}=\frac{\hat{\boldsymbol{w}}^{\prime} \mathbf{X}^{\prime} \mathbf{X} \hat{\boldsymbol{w}}}{\alpha(1-\alpha)} \sim \chi^{2}(M+2)
$$

In the empirical application, we follow Engle and Manganelli (2004) to explore the dynamic quantile test: DQ in which $\mathbf{X}$ contains a constant, four lagged hits, $H_{t-1}(\alpha), \cdots, H_{t-4}(\alpha)$, and a contemporaneous VaR estimate, $q_{t}(\alpha)$.

It should be mentioned that there are a large number of VaR backtesting methods for the detection of high-order independence, such as durationbased test (Christoffersen and Pelletier, 2004) and martingale difference test (Berkowitz et al., 2005), whereas the DQ test of Engle and Manganelli (2004) performs best overall with either simulated data or real market data (Berkowitz et al., 2007). 


\section{Autocorrelation test}

From $E\left[\left(I_{t+1}-\alpha\right)\left(I_{t-k}-\alpha\right)\right]=0$, we can construct an autocorrelation test as

$$
H_{0}: \gamma_{k}=0, k>0,
$$

where $\gamma_{k}$ is the autocorrelation between $I_{t+1}-\alpha$ and $I_{t-k}-\alpha$. A natural statistic is the Ljung-Box statistic such that

$$
L B(m)=T(T+2) \sum_{k=1}^{m} \frac{\gamma_{k}^{2}}{T-k} \longrightarrow \chi^{2}(m) \text { (asymptotically) }
$$

\section{Duration test}

Christoffersen and Pelletier (2004) formulate the duration-based backtest as follows. The durations for the test statistic are defined as the times (in days) between two VaR violations:

$$
D_{i}=t_{i}-t_{i-1}
$$

where $t_{i}$ denotes the date when the $i$-th violation occurs. Under independence, the mean duration is $1 / \alpha$ and the duration function must have no memory. Christoffersen and Pelletier (2004) suggest the hazard function of the duration distribution under the null hypothesis to be the exponential function because it is continuous and memory-free. For the hazard function in the alternative hypothesis, they choose the hazard of the Weibull distribution. The hazard function of the Weibull distribution has a closed-form representation:

$$
\lambda_{W}(D)=a^{b} b D^{b-1} .
$$

The hazard of the exponential function is a special case of the hazard of the Weibull distribution when $b=1$. Thus, it is possible to test the independence by defining the null hypothesis as:

$$
H_{0, \text { ind }}: \quad b=1 \text {, }
$$

and the log-likelihood function is

$$
\begin{array}{r}
\ln L\left(D \mid \Omega_{T}\right)=C_{1} \ln S\left(D_{1}\right)+\left(1-C_{1}\right) \ln f\left(D_{1}\right)+\sum_{i=2}^{N(T)-1} \ln \left(f\left(D_{i}\right)\right) \\
+C_{N(T)} \ln S\left(D_{N(T)}\right)+\left(1-C_{N(T)} \ln f\left(D_{N(T)}\right)\right),
\end{array}
$$


where $f(\cdot)$ is the hypothesis specific probability distribution function (PDF), $S(\cdot)$ is its survival function, $D_{i}$ is the duration of the $i$-th $\mathrm{VaR}$ violation, $T$ is the total number of violations in the sequences and $C_{1}$ and $C_{N(T)}$ are binomial censor indicators for the first and last duration, respectively. The PDF can be, for example, the exponential function in the case of the null Weibull hypothesis. The censor variables $C_{1}$ and $C_{T}$ indicate whether the series of violations begins or ends with a violation. If the violation sequence begins with 0 then $C_{1}=1$, and $D_{1}$ is the duration until the first violation. If the violation sequence begin with 1 then $C_{1}=0$, and $D_{1}$ is the duration until the second violation. If the violation sequence ends with 0 then $C_{N(T)}=1$, and $D_{N(T)}$ is the number of days after the last violation. If the violation sequence ends with 1 then $C_{T}=0$, and $D_{N(T)}$ is the duration of the last violation. Thus, the likelihood ratio test statistic for the test of independence is

$$
L R_{\text {ind }}=2\left[\ln L\left(D \mid \Omega_{T}\right)-\ln L\left(\hat{D} \mid \Omega_{T}\right)\right]
$$

\section{References}

Artzner, P., F. Delbaen, J.M. Eber, and D. Heath (1999): "Coherence Measures of Risk." Mathematical Finance 9, 203-228.

Bao, Y., T. Lee, and B. Saltoğlu (2006): "Evaluating the Predictive Performance of Value-at-Risk Models in Emerging Markets: A Reality Check." Journal of Forecasting 25, 101-128.

Bertail, P., C. Haefke, D.N. Politis, and H. White (2004): "A Subsampling Approach to Estimating the Distribution of Diverging Statistics with Application to Assessing Financial Market Risk." Journal of Econometrics 120, 295-326.

Berkowitz, J., P.F. Christoffersen, and D. Pelletier (2009): "Evaluating Valueat-Risk Models with Desk-Level Data." Forthcoming in Management Science.

Christoffersen, P.F. (1998): "Evaluating Interval Forecasts." International Economic Review 39(4), 841-862.

Christoffersen, P.F. (2008): "Backtesting." Encyclopedia of Quantitative Finance, R. Cont(ed). John Wiley and Sons. 
Danielsson, J. and Y. Morimoto (2000): "Forecasting Extreme Financial Risk: A Critical Analysis of Practical Methods for the Japanese Market." Monetary and Economic Studies 18(2), 25-48.

Engle, R.F. and S. Manganelli (2004): "CAViaR: Conditional Autoregressive Value at Risk by Regression Quantiles." Journal of Business and Economic Statistics 22, 367-381.

Fama, E. (1965): "The Behavior of Stock Prices." Journal of Business 38(1), 34-105.

Guidolin, M. and A. Timmermann (2006): "Term Structure of Risk under Alternative Econometric Specifications." Journal of Econometrics 131, 285-308.

Komunjer, I. (2004): "Quasi-Maximum Likelihood Estimation for Conditional Quantile." Journal of Econometrics 128, 137-164.

Kouretas, G.P. and L. Zarangas (2005): "Conditional Autoregressive Valueat-Risk by Regression Quantiles: Estimation Market Risk for Major Stock Markets." Working paper, available from http://econ.uop.gr/ econsem/index_files/CAViaR1.pdf.

Kuester, K., S. Mittnik, and M.S. Paolella (2006): "Value-at-Risk Prediction: A Comparison of Alternative Strategies." Journal of Financial Econometrics 4(1), 53-89.

Kupiec, P. (1995): "Techniques for Verifying the Accuracy of Risk Measurement Models." Journal of Derivatives 3, 73-84.

Li, M.-Y.L. and H.-W.W. Lin (2004): "Estimating Value-at-Risk via Markov Switching ARCH Models - An Empirical Study on Stock Index Returns." Applied Economics Letters 11(11), 679-691.

Mandelbrot, B.B. (1963): "The Variation of Certain Speculative Prices." Journal of Business 36(2), 394-419.

Manganelli, S. and R.F. Engle (2004): "A Comparison of Value-at-Risk Models in Finance." in G. Szego (eds.), Risk Measures for the 21st Century, Chichester, UK: Wiley: 123-143.

Venkataraman, S. (1997): "Value-at-Risk for a Mixture of Nomal Distributions: The Use of Quasi-Bayesian Estimation Techniques." Economic Perspectives 21(2), 2-13. 